\title{
RADIOLOGICAL FINDINGS IN THE LUNGS OF PREMATURE INFANTS
}

\author{
BY \\ JOHIN FAWCITT \\ From Crumpsall Hospital, Manchester
}

(RECEIVTD FOR PUBLICATION NOVEMBER 2, 1955)

What is the place of the rad:ologist in the diagnosis and treatment of the ailments of the premature infant? First, he must be able to recognize the variations in the normal premature baby's chest and abdomen. Secondly, he must attempt to give the clinician some idea of the identity of the pathological changes in the lungs. Thirdly, and probably his most important function, is to try and help the paediatrician to distinguish between the respiratory distress which is primarily cerebral in origin and that due directly to pathological changes in the lungs.

These infants cannot readily be moved down to the main $x$-ray department in the average hospital in this country, nor is it advisable to do so. We, therefore, use a standard mobile $x$-ray apparatus with a minimum time exposure factor of 0.05 seconds, which is kept permanently in the premature babies' ward, and as soon as the infant arrives in the ward it is laid on a casette in the incubator and a radiograph of the chest and abdomen taken. The initial radiograph may be the only one, for it has been considered that no further films are justified in an infant who exhibits no significant respiratory distress and is thriving. If, however, there is any obvious alteration in the clinical progress, radiographs are taken at appropriate intervals.

Using the methods described, a surprisingly high percentage of the films taken have been of very good quality, and few have been useless for diagnostic purposes. It is fully realized that the technique is far from ideal, but it has been considered that any radiograph was better than none at all.

The advisability of disturbing the premature infant to carry out this procedure may well be questioned, but it was felt that the chance of arousing intercurrent infection was slight, that the amount of disturbance to the infant was not significant, and that the degree of radiation was negligible.

Certain variables and artefacts make the interpretation of these radiographs far from simple. The phase of the respiratory cycle makes a quite extraordinary difference in the apparent degree of aeration of the lung fields, and the phase of contraction of the heart also alters the pattern to a considerable degree. These appear to have a greater variant effect in the first few hours of life than at a slightly later stage. Another artefact which may show is the presence of vertical lines over the lung fields which may simulate a pneumothorax under certain conditions. These are due to the skin folds caused by the lack of subcutaneous fat in the premature infant.

The displacement $o$ the heart to the left, which has been seen in a fairly high percentage of these cases, is in my opinion due to the plasticity of the thoracic cage and the consequent sternal suction inwards.

During the last 18 months 190 infants have been examined by this radiographic technique in the premature babies' ward at Crumpsall Hospital, Manchester. The great majority were delivered in the Hospital, but a few came in from the District within an hour or two of birth.

Forty-nine of these did not survive, and the cause of their deaths is shown in Table 1. Of the remaining cases, 25 showed pathological changes in the

\section{TABLE 1}

CAUSE OF DEATH IN 49 PREMATURE INFANTS

Primary lung unexpansion from all causes, including prematurity

Miscellaneous causes

(i) Associated congenital anomalies

(ii) Unexplained

(iii) Unexplained

Cerebral deaths with lung expansion

Hyaline membrane

Neonatal pneumonia

Sclerema

Haemorrhagic disease of the newborn

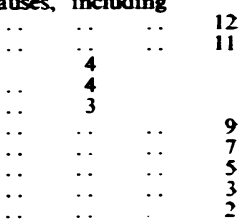

radiographs of the lungs and clinical respiratory distress (Table 2).

It has been stated (Martin and Freidell, 1952) that the lungs of the newborn do not become fully expanded for up to six days and that primary atelectasis may be shown histologically for that 
period in full-term infants, whilst it may take up to six weeks for the premature infant fully to expand the lungs.

TABLE 2

PATHOLOGICAL CHANGES IN 25 INFANTS

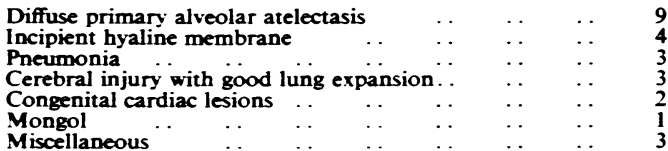

I have taken serial radiographs of a number of full-term children at caesarean section from eight seconds to 12 minutes after the cutting of the cord, and have found that there is complete expansion of the lungs radiologically-in the majority by six minutes. I consider that this rapid adequate aeration is of far greater practical significance than the microscopical unexpansion of small areas of lung.

A different state of affairs obtains in the radiographs of lungs of premature infants, in whom I have observed that practically every chest examined radiologically within an hour of birth showed some fairly well marked areas of unexpansion. These were mainly in the lower lobes, and were undoubtedly more marked on the left than on the right side. In uncomplicated cases these areas showed fairly complete expansion within six to eight hours.

\section{Pulmonary Conditions in Present Series}

Pulmonary conditions observed in the present series may be broadly divided into the following categories:

(a) Prematurity or immaturity, leading to complete or almost complete primary atelectasis:

(b) Intracranial lesions resulting in primary unexpansion of the lungs:

(c) Intracranial lesions allowing expansion of the lungs initially;

(d) Primary lung lesions

(i) Hyaline membrane

(ii) Neonatal pneumonias

(iii) Diffuse primary alveolar atelectasis

(iv) Haemorrhagic disease of the newborn

(e) Congenital cardiac lesions. (These have not been specifically studied in this series.)

(a) Prematurity or Immaturity. Plain uncomplicated immaturity (described by the coined word 'anectatos', Farber and Wilson, 1933), may lead to unexpansion of the lungs for a variety of interrelated reasons. Of these the major considerations appear to be the immaturity of the lungs, the poverty of the respiratory effort and the excessive plasticity of the thoracic cage.

This type of unexpansion follows the pattern of primary atelectasis or unexpansion, which is seen in other forms of this catastrophe, and can only be distinguished from them by the maturity of the infant and knowledge of the time after birth that the radiograph had been taken. The larger bronchi are clearly outlined against the practically opaque lung parenchyma. The outlines of the heart and diaphragms are hazy and the cardiac shadow is often displaced to the left. A histological study of these lungs generally shows terminal anoxic haemorrhages into many of the alveoli with large areas of alveoli which have never been aerated, showing cubical rather than pavement epithelium lining them.

Bowel gas shows sluggish motility and the infant practically never survives.

(b) Intracranial Lesions Resulting in Primary Unexpansion of the Lungs. There are types of intracranial injury which occur during parturition which are so widespread or devastating that the infants' respiratory centre is severely damaged at that time. They do not make adequate respiratory efforts, and, like those who die of their prematurity, the lungs never become adequately expanded. The radiological appearances are not distinguishable from those of grossly premature infants except by their greater bone maturity and size. The bowel gas progresses poorly, though this picture may be falsified by the use of intragastric oxygen.

(c) Intracranial Lesions Allowing Initial Expansion of Lungs. In general, tentorial tears and intraventricular haemorrhages are progressive in their effects upon the respiratory centre, and there is reasonable expansion of the lungs initially in most of these cases.

With increasing damage to the brain from haemorrhage and with failing respiratory effort, areas of atelectasis become apparent in the radiographs. These do not take on the miliary pattern of hyaline membrane and there is not usually enough atelectasis in the lung fields to suggest a pure respiratory death, except in radiographs taken in the completely terminal stages. There the familiar pattern of the 'blacked-out' lung is seen with its opaque lung fields, vanished cardiac silhouette and obliterated diaphragmatic outlines.

The guiding radiological feature in the diagnosis of progressive intracranial damage is, in my opinion, the discrepancy between the relatively slight degree of atelectasis in the lung fields and the severity of the respiratory distress. 
(d) Primary Lung Lesions. These fall into four classes.

(i) Pulmonary Hyaline Membrane. Hoccheim described this condition first in 1903 . The radiological appearances have not received much attention until recently, when they were fully described by Steiner (1954) in this country and by a number of other authors, mainly in the United States.

The cases in this series conform well to the pattern described by Steiner. There is some unexpansion at birth, but fair respiratory effort. This is followed by a miliary mottling, which tends to be fairly widespread but may be somewhat localized. These changes may be present in the early radiographs, but always manifest themselves within 12 hours of birth. As the disease progresses the mottling takes on a coarser and more generalized appearance, followed by atelectasis of a blotchy type. Eventually, after a period of increasing collapse and consolidation, the blacked-out lung of the terminal stage makes its appearance.

The clinical condition keeps step with the radiological findings, and the infant rarely survives more than 30 hours.

There is another radiological pattern which may be suspected as incipient hyaline membrane. In this, the lung fields show the miliary mottling previously described, and in which they may go on to show a coarser mottling, but do not go beyond the phase of minor lobular atelectasis. As the clinical condition improves the mottling slowly fades, but cases have been seen in which it has persisted for six to seven days.

Histological proof that this is indeed the hyaline membrane syndrome is of course missing, but, as Donald and Steiner (1953) point out, the clinical features tie up very closely with the earlier manifestations of the known fatal type of hyaline membrane.

(ii) Neonatal Pneumonias. I do not think that it is possible to distinguish between the pneumonias of the immediate neonatal period, whether they be due to metastatic infection from the maternal circulation, inhaled infected liquor during parturition, or post-natal infective processes. In the present series there were nine cases of what was considered to be pneumonia. Six of these were proven at necropsy, and three of them survived, but clinically and radiologically were typical of the condition.

The common pattern was that of a bronchopneumonia radiologically, but two showed a confluent lobar pattern which involved the right upper lobe. (iii) Diffuse Primary Alveolar Atelectasis. There is another group of infants, some of whom survive, the radiographs of whose lung fields show a ground glass appearance which is evenly distributed throughout. The pattern is not that of hyaline membrane, and even in those who do not live does not develop into the coarse mottling and type of atelectasis associated with that condition. This radiological appearance is associated with some degree of respiratory distress which is usually surmounted. When the infant has not survived, the post-mortem findings in the lungs have been those of considerable areas of primary alveolar atelectasis histologically, and an absence of hyaline membrane.

I have observed this condition radiologically as early as seven minutes after birth, when it was already distinguishable from the normal at that age. It is possible, in my opinion, to distinguish it from hyaline membrane, and thus give a more hopeful prognosis.

This condition appears to be a less severe form of primary alveolar atelectasis, and I would, therefore, describe it as diffuse primary alveolar atelectasis.

(iv) Haemorrhagic Disease of the Newborn. Only two cases of this condition were radiographed in the present series, both of which failed to survive. In the first, a rather confident radiological diagnosis of pulmonary hyaline membrane was made. In the second, and without clinical information, it was suggested that the appearances in the radiograph were not entirely typical of hyaline membrane but might be those of haemorrhagic disease of the newborn.

The radiological picture differed only very slightly in the two conditions. The small areas of atelectasis causing the miliary mottling in the lung fields seemed rather larger on the whole than those of hyaline membrane, and appeared rather more discrete. It was felt, however, that the distinction was a very fine one and that it was not possible to separate the radiological appearances of the two conditions with any confidence.

(e) Congenital Cardiac Lesions. No attempt was made specifically to investigate the congenital cardiac lesions or other congenital anomalies observed in this series.

\section{Clinical Cases}

It must be realized that the radiological patterns in the neonatal lung are generally so delicate that it is not possible adequately to describe them in a paper; particularly is this true of the diffuse primary alveolar atelectasis. Therefore only examples of those conditions showing distinctive changes will be singled out for demonstration. 
Immaturity. The following case illustrates the pattern found in immaturity.

J. G., a boy, of 25 weeks' maturity (weight $1 \mathrm{lb}$. $2 \frac{1}{2} \mathrm{oz}$.) was the first child of his mother from whom no obstetric history of note was obtained, and whose labour was uncomplicated.

The infant's condition was poor, but respiration was regular, and he gave a few squeaky cries. His condition steadily deteriorated and death took place three and a quarter hours after birth.

Radiological Findings. At one and a half hours (Fig. 1) there was primary atelectasis of most of both

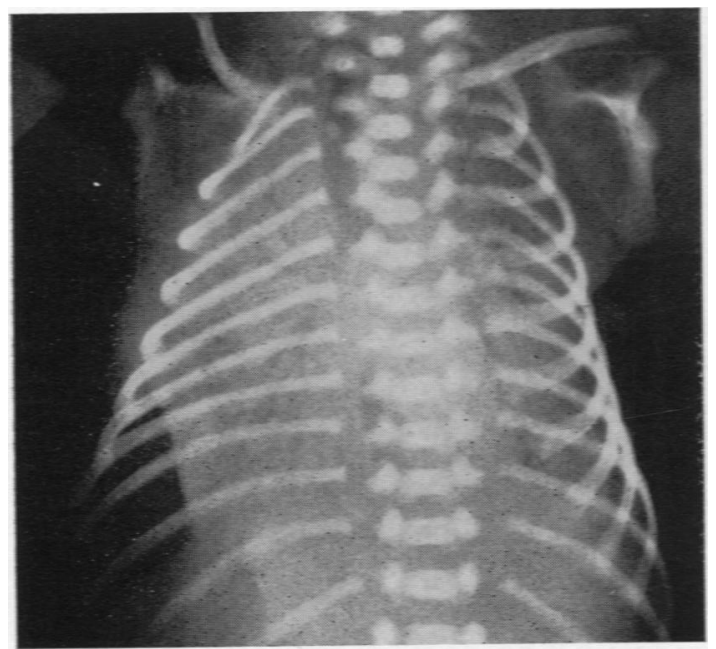

Fig. 1.-Primary atelectasis of most of both lung fields, with the main and secondary bronchi outlined against the semi-opaque lung tissue.

lung fields with the main and secondary bronchi outlined against the semi-opaque lung tissue. The diaphragmatic and cardiac outlines were poorly defined. At three hours a film was taken as the baby was in extremis. The blacked-out lung fields were shown.

POST-Mortem Findings. There were no significant findings except in the lungs, which, though pink, sank in water.

Histological Findings. In the right upper lobe very little aeration had occurred, and the features were those of primary non-expansion: no membrane.

The right lower and left upper lobes had not expanded primarily; no membrane. In the left lower lobe there were intra-alveolar haemorrhages and the lobes had not expanded primarily; no membrane.

Hyaline Membrane. The case of D.W., a girl of 36 weeks' maturity (4 lb. $4 \mathrm{oz}$.), illustrates the findings with hyaline membrane.

The mother had had six pregnancies with one stillbirth. There was an obstetric history of A.P.H. at 30 weeks and labour lasted one hour 35 minutes. The mother was febrile in labour, and the placenta was unhealthy.

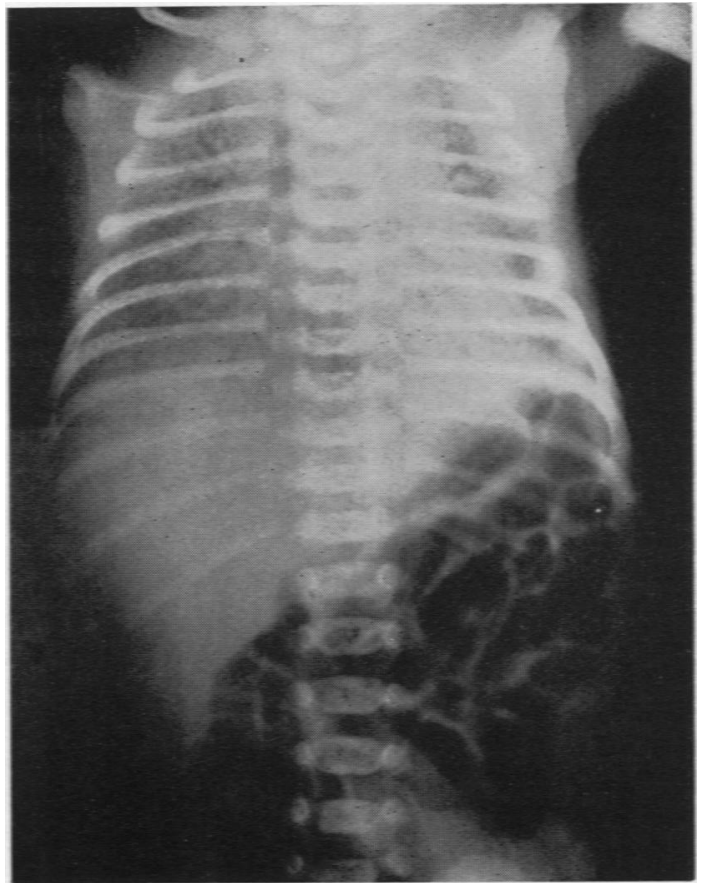

Fig. 2.-Coarse mottling and greatly increased atelectasis, and bowel gas only in the ileum.

The infant was pale and shocked with limited air entry into the lungs. The sternum was sucked in and cyanosis steadily deepened until death at seven hours and 50 minutes after birth.

Radiological Findings. At 35 minutes there was a fair degree of unexpansion with a fair mottling throughout the lung fields, changes suggestive of hyaline membrane.

Gas in the bowel progressed onward relatively poorly. At 3 hours mottling had increased with further atelectasis. At 6 hours 40 minutes (Fig. 2) there was coarser mottling with greatly increased atelectasis, and bowel gas only in the ileum. At 7 hours $\mathbf{5 0}$ minutes the blacked-out lung of death was seen.

POST-MORTEM FIndings. Pathology was limited to the lungs in which there was considerable secondary atelectasis and well marked hyaline membrane. There were residual areas of primary atelectasis.

Neonatal Pneumonia. The following case illustrates the findings in neonatal pneumonia.

D.B., a male twin, of 37 weeks' maturity (4 lb. 4 oz.) was born of a mother who had already had one pregnancy. There was no obstetric history of note and labour was uncomplicated.

The infant's condition was good and the lung expanded satisfactorily for the first two days after birth, after which he collapsed with convulsions and occasional gasping inspirations, dying some 12 hours later. 
Radological Findings. At 1 hour 20 minutes there was satisfactory expansion of both lung fields, with the slight unexpansion one might expect in a premature infant at that stage. At 2 days there was still good expansion of the lung fields. Intragastric oxygen was being given. At 2 days 12 hours (Fig. 3) a film was taken almost at the point of death. There was con-

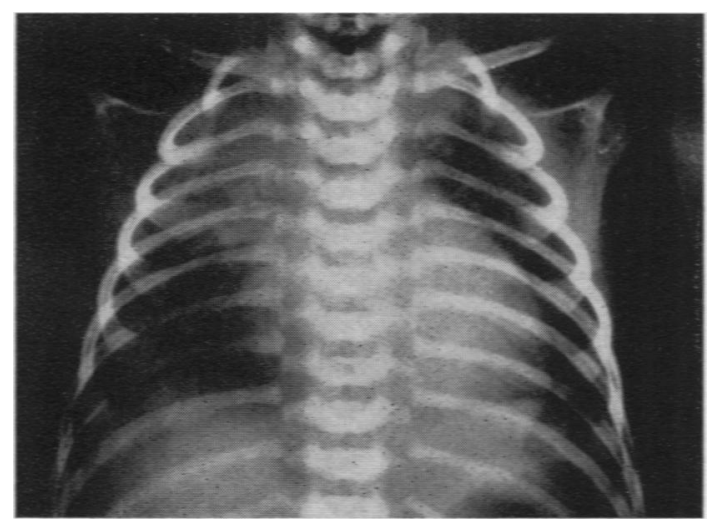

Fig. 3.-Film taken at the point of death.

solidation of the right upper lobe and some atelectatic changes of the right lower lobe. There were also pneumonic changes in the left upper lobe.

POST-MORTEM FInDINGs. Pathology was limited to the lungs, which showed pneumonic consolidation of the right upper lobe and the adjacent part of the lower lobe.

Histological Findings. The alveoli were filled with polymorphs and the bronchioles contained polymorphs as well. There were some amniotic squames. The appearances were those of a confluent bronchopneumonia.

\section{Conctusions}

The radiographs of 190 premature infants have been examined in the present series, and it is considered that this ancillary investigation may help the clinician considerably in his conduct of the cases.

From this limited preliminary survey I think that it is possible to distinguish radiologically between the acute respiratory distress due to an intracranial lesion and that of a pulmonary condition.

The radiological appearances of pulmonary hyaline membrane are fairly clearly defined, but may be closely simulated by those of haemorrhagic disease of the newborn involving the lung and certain phases of neonatal pneumonia.

The condition I have termed diffuse primary alveolar atelectasis' appears to me to be generally distinguishable from hyaline membrane, and to have a more favourable ultimate prognosis but further investigation is necessary on this point.

I would like to thank Dr. Patterson, who is in charge of the Premature Babies' Ward at Crumpsall Hospital, for his stimulation to carry out this investigation and his help in doing so. I would also like to thank Dr. Davson for his interest and cooperation in correlating the histological and radiological pictures.

Finally, I would especially thank Sisters Thornley and Maclaren, who have been responsible for taking the majority of the radiographs and whose interest and help in this project have been invaluable.

\section{REFERENCES}

Donald, I. and Steiner, R. E. (1953). Lancet, 2, 846.

Farber, S. and Wilson, J. L. (1933). Amer. J. Dis. Child., 46, 572. Martin, J. F. and Friedell, H. L. (1952). Amer. J. Roentgenol., $67,905$.

Steiner, R. E. (1954). Brit. J. Radiol., 27, 491. 